\title{
Open future, supervaluationism and the growing-block theory: a stage-theoretical account
}

\section{Roberto Loss ${ }^{1}$ (D)}

Received: 28 March 2021 / Accepted: 12 September 2021

(c) The Author(s), under exclusive licence to Springer Nature B.V. 2021, corrected publication 2022

\begin{abstract}
I present a 'stage-theoretical' interpretation of the supervaluationist semantics for the growing-block theory of time according to which the 'nodes' on the branching tree of historical possibilities are taken to be possible stages of the growth of the growingblock. As I will argue, the resulting interpretation (i) is very intuitive, (ii) can easily ward off an objection to supervaluationist treatments of the growing-block theory presented by Fabrice Correia and Sven Rosenkranz, and (iii) is also not saddled by the problems affecting the supervaluationist version of the growing-block theory defended by R. A. Briggs and Graeme A. Forbes.
\end{abstract}

Keywords Open future $\cdot$ Supervaluationism · Growing-block theory $\cdot$ Temporal passage $\cdot$ A-theory $\cdot$ Future contingents $\cdot$ Truth-value gaps $\cdot$ Indeterminacy

\section{Introduction}

The growing-block theory belongs to the family of A-theoretic, or 'dynamic', theories of time. Growing-block theorists take temporal passage to consist in the fact that the 'sum total of existence is always increasing' (Broad, 1923: p. 66). According to them, the 'block' of reality comprises both past and present entities and grows as time passes, while the present time is just the latest time that has come into existence. One of the most important features of the growing-block theory is the kind of stark ontological asymmetry it posits between the past and the future, which allows growing-block theorists to give a simple and straightforward account of the intuition that the future is 'open' while the past is 'settled'. ' In turn, at least under certain assumptions concerning

\footnotetext{
${ }^{1}$ See Grandjean (2019) for some recent discussion on how to characterize the asymmetry between the past and the future.
}

This article belongs to the topical collection "Indeterminacy and Underdetermination", edited by Mark Bowker and Maria Baghramian.

\footnotetext{
$\bowtie$ Roberto Loss

robertoloss@gmail.com

1 Department of Logic and Theoretical Philosophy, Complutense University of Madrid, Madrid, Spain
} 
the way truth is grounded in reality, the non-existence of the future suggests that historically contingent statements about the future (the so-called 'future contingents') lack a truth-value and are therefore neither true nor false. One of the most promising semantics for future-contingent statements is Thomason's (1970) supervaluationism. Roughly, according to this approach, a statement $S$ is true (or 'super-true') if it is true according to every way the future might turn out to be (every 'possible future'), false (or 'super-false') if it is false according to every possible future, and neither true nor false otherwise.

In this paper I will not defend either the idea that growing-block theorists should take future-contingent statements to be neither true nor false nor the idea that supervaluationism is the best semantic treatment for 'gappy' future-contingents. ${ }^{2}$ Instead, the aim of this paper is to argue that growing-block theorists employing Thomason's supervaluationist semantics should interpret it 'stage-theoretically' and take the entities that in supervaluationist models are ordered in a tree-like structure to represent possible 'stages' of the growth of the growing-block. As I will argue, the resulting interpretation of the supervaluationist machinery (i) is rather intuitive, (ii) can be used to resist an objection to supervaluationist growing-block theories recently advanced by Correia and Rosenkranz (2018: p. 105), and (iii) is also not saddled with the problems surrounding one of the most prominent supervaluationist accounts of the growingblock theory on the market, namely the one presented by Briggs and Forbes (2012).

\section{Stage setting}

\subsection{The growing-block theory}

I will take the growing-block theory to be a dynamic, 'A-theory' of time that is characterized by two main claims, which can be found in one of the earliest formulations of the theory due to C. D. Broad $(1923)^{3}$ :

(BR1) 'The sum total of existence is always increasing [...]. (Broad, 1923: pp. 66-67)

(BR2) 'There is no such thing as ceasing to exist; what has become exists henceforth forever.' (Broad, 1923: p. 69) ${ }^{4}$

Furthermore, I will assume that the A-theoretical nature of the growing-block theory consists in the idea that the most 'metaphysically perspicuous' truths concerning the dynamic nature of time are expressed by means of the irreducible, 'joint-carving' temporal operators 'it was the case that' (' $P$ ') and 'it will be the case that' (' $F$ ')—where the operators 'it was always the case that' ('H'), 'it will always be the case that' (' $G$ '),

\footnotetext{
2 Correia and Rosenkranz (2018: chapter 7) argue that the growing-block theory is compatible with both the principle of bivalence and the idea that truth is (somehow) grounded in reality. For alternative semantics for growing-block theorists who reject the principle of bivalence, see Briggs and Forbes (2012: pp. 283-288).

3 Thomas (2019) argues that the growing-block theory was firstly articulated by the British emergentist Samuel Alexander.

4 See Correia and Rosenkranz (2018: p. 36) on this point.
} 
and 'it is always the case that' (' $A$ ') can instead be defined, as it is customary, ${ }^{5}$ as follows:

$$
H p=_{d f} \sim P \sim p \quad G p=_{d f} \sim F \sim p \quad A p=_{d f} p \wedge H p \wedge G p
$$

This means that letting ' $\mathbb{E}$ ' stand for the existence predicate

$$
\mathbb{E} x={ }_{d f} \exists y(x=y)
$$

the two characterizing features of the growing-block theory just reviewed above can be formalized as follows:

$$
\text { (GB1) } A \exists x \sim P \mathbb{E} x
$$

\section{(GB2) $A \forall x G \mathbb{E} x$}

(GB1) says that it is always the case that something never existed before (so that it is true that 'sum total of existence is always increasing'), while (GB2) says that it is always the case that everything that exists will always exist in the future (so that it is true that 'what has become exists henceforth forever').

In what follows I will not defend this characterization of the growing-block theory. Notice, however, that both the idea of (i) taking (BR1) and (BR2) to be two core features of the growing-block theory of time and the idea of (ii) characterising Atheories of time in general as employing irreducible tense operators appear to be rather common in the literature (see, among others: Sider, 2011: pp. 263-264; Smith, 2011: p. 241; Sullivan, 2012: p. 150; Pooley, 2013: p. 333; Tallant \& Ingram, 2015: p. 356; Skow 2015: pp. 52-58; Deasy, 2017: p. 391; Correia \& Rosenkranz, 2018: pp. 36-50; Loss, 2019: pp. 1136-1137). Notice, furthermore, that in this paper I will not give a full-fledged account of the growing-block theory, but will only be concerned with its central ontological claims (like GB1 and GB2). At the same time, I will try to be as ecumenical as possible and leave open other important issues surrounding the theory (like, for instance, the so-called 'present problem'). ${ }^{6}$

\subsection{Thomason's supervaluationism}

Thomason's (1970) supervaluationist approach to future-contingent statements employs a set $K$ of entities, which Thomason calls 'times', ordered in a tree-like

\footnotetext{
5 See Goranko and Rumberg (2020: §3.1).

${ }^{6}$ For an introduction to the 'present problem' see, among others, Bourne (2002), Merricks (2006), Correia and Rosenkranz (2013) and Braddon-Mitchell (2004, 2013). For some defence of the idea that the past is 'real but dead' see Forrest (2004) and Forbes (2016). Notice that the idea that the past may be 'dead' in the relevant sense (so that that past people like, say, Marie Curie are now completely devoid of conscious thought) is compatible with the characterisation of the growing-block theory I am assuming in this paper only if the 'dying' of the past doesn't involve any ontological loss, so that even if Marie Curie isn't conscious anymore, it is nevertheless false that there was something (like, say, 'Marie Curie's consciousness') that doesn't exist anymore.
} 
fashion by a relation ' $<$ '. 7 A history is a maximal linear chain of times. Therefore, a model of Thomason's supervaluationism looks like a 'branching tree' of histories and is such that, for each point $t$ on the tree, there is a plurality of histories overlapping towards 'the past' of $t$ and branching towards 'its future' intuitively representing all the possible futures of $t$. The gist of Thomason's semantics can be presented in the propositional case along the following lines. First, we define a notion of truth that is relative to both a time and a history as follows (where $V$ is a usual valuation function from the set of times to the power set of the set of atomic sentences):

$$
\begin{gathered}
\langle t, h\rangle \vDash p \Leftrightarrow p \in V(t) \quad \text { (if } p \text { is atomic) } \\
\langle t, h\rangle \vDash p \wedge q \Leftrightarrow\langle t, h\rangle \vDash p \text { and }\langle t, h\rangle \vDash q \\
\langle t, h\rangle \vDash \sim p \Leftrightarrow\langle t, h\rangle \not \models p \\
\langle t, h\rangle \vDash F p \Leftrightarrow \exists s[s \in K \wedge t<s \wedge s \in h \wedge\langle s, h\rangle \vDash p] \\
\langle t, h\rangle \vDash P p \Leftrightarrow \exists s[s \in K \wedge s<t \wedge s \in h \wedge\langle s, h\rangle \vDash p]
\end{gathered}
$$

Then, the notions of truth and falsity (or 'super-truth' and 'super-falsity') at a time are defined by 'quantifying out' the history parameter, as follows ${ }^{8}$ :

$$
\begin{aligned}
& \operatorname{True}(p, t) \Leftrightarrow \forall h[t \in h \rightarrow\langle t, h\rangle \vDash p] \\
& \operatorname{False}(p, t) \Leftrightarrow \forall h[t \in h \rightarrow\langle t, h\rangle \not \models p]
\end{aligned}
$$

In other words, according to this semantics, a sentence is (super-)true with respect to a time if and only if it is true at that time with respect to all the histories 'passing through' that time, and it is (super-)false with respect to a time if and only if it is false at that time with respect to all those histories. Instead, when a sentence is true at a time only with respect to some histories and false with respect to others, then it is neither true nor false at that time. This is precisely what happens in the case of future contingent statements. Consider, for instance, the following toy model. We have three times, $\mathrm{T} 1, \mathrm{~T} 2$, and $\mathrm{T} 3$, and they are such that $\mathrm{T} 1<\mathrm{T} 2, \mathrm{~T} 1<\mathrm{T} 3$, but it is neither the case that $\mathrm{T} 2<\mathrm{T} 3$ nor that $\mathrm{T} 3<\mathrm{T} 2$ (so that $\mathrm{T} 1$ is represented as preceding both $\mathrm{T} 2$ and T3 which are times that don't belong to the same history). H1 is the history $<\mathrm{T} 1$, $\mathrm{T} 2>$, while $\mathrm{H} 2$ is the history $<\mathrm{T} 1, \mathrm{~T} 3>$. Therefore, $\mathrm{T} 2$ and $\mathrm{T} 3$ represent two possible futures of T1. Suppose that $V(p)=\{\mathrm{T} 3\}$, so that the atomic sentence $p$ is true only

\footnotetext{
${ }^{7}$ More precisely, $<$ is irreflexive, asymmetric, transitive, connected, and 'left-linear' (that is, such that, for every $x, y$ and $z$, if $x<y$ and $z<y$, then either $x<z, z<x$, or $x=z$ ).

${ }^{8}$ See Belnap (2009) for a critical discussion on supervaluationism and the way it 'quantifies out' auxiliary parameters.
} 
with respect to time T3. ${ }^{9}$ It follows that the sentence ' $F p$ ' ('it will be the case that $p$ ') is true with respect to $\langle\mathrm{T} 1, \mathrm{H} 2\rangle$ but false with respect to $<\mathrm{T} 1, \mathrm{H} 1>$. As a matter of fact, it is true that the time $t$ that is later than T1 according to H2 (namely, T3) is such that $p$ is true at $t$. However, the time $t$ that is later than T1 according to H1 (namely, $\mathrm{T} 2$ ) is such that $p$ is false at $t$. Therefore, we have that ' $F p$ ' is true only according to some of the histories passing through $\mathrm{T} 1$ and is thus neither true nor false at $\mathrm{T} 1$.

\section{All the stages of temporal passage}

Central to the 'stage-theoretical' account of supervaluationist growing-block theories that I will be defending in this paper is the idea of taking Thomason's 'times' to be a specific subset of the familiar ersatz possible worlds that can be employed also by eternalist B-theorists for the semantics of modal statements, ${ }^{10}$ and namely, ersatz worlds that represent the block of reality as ending at a certain time. Given the general purpose of this paper we can ignore the details of the kind of ersatzism that may be adopted and let growing-block theorists make their specific choices according to their philosophical preferences. ${ }^{11}$ What is more important is what these ersatz possible worlds are meant to represent in this framework, and namely, all the possible 'stages' of the growth of the growing block of reality. The idea, in a nutshell, is the following. Assume that the actual universe begins with the Big Bang. Since we are also assuming that the future is genuinely open and indeterminate, there are many ways in which the block may grow after the Big Bang takes place. According to each way, the block gets bigger and bigger as newer and newer entities come into existence. A possible 'stage' of the growth of the block in this sense is thus just a possible 'moment' of the growth of the block according to one of the possible ways in which the block of reality can grow given the growing-block theory at hand (this notion of 'stage' must thus not to be confused with the notion of 'stage' featuring the theory of persistence known as 'stage theory'). ${ }^{12}$ For instance, there is a possible stage in which the block is as it is right now (that 2021 is present) and thus such that some time $t$ in 2021 is the latest time on the block (we can call this stage the 'actual' stage). But there is also a possible stage in which the block is such that 2021 is present, but in which the Covid-19 pandemic was successfully contained and life returned pretty much to normal by December 2020. We can imagine that these two possible stages agree on what happens up until, say, March 2020 and disagree on the kind of response that governments throughout the world have given to the Covid-19 crisis. In addition to these two possible stages, there

\footnotetext{
${ }^{9}$ It should be noticed that this notion of truth-at-a-time- $t$ (corresponding to the fact that the sentence in question is a member of $V(t))$ is different from both the supervaluationist notion of super-truth a time and the notion of truth-at-a-time-and-a-history. For simplicity's sake I employ 'truth at' in every case and let the context disambiguate.

10 See Menzel (2017: §2.2) for an overview of ersatzism in general (there labelled 'abstractionism'). See also Bourne (2006: pp. 52-61) and Crisp (2007: pp. 98-105) for two ersatzist approaches to presentism.

11 For instance, growing-block theorists may take ersatz possible worlds to be maximal and consistent sets of propositions not featuring the irreducible tense operators they employ to express facts about the growth of the block (so that < Barak Obama is the president of the USA in $2009>$ counts as a proposition of the right kind but < It will be the case that: Kamala Harris is the president of the USA in $2025>$ doesn't).

12 On the theory of persistence known as 'stage theory' see Sider (2001) and Hawley (2001).
} 


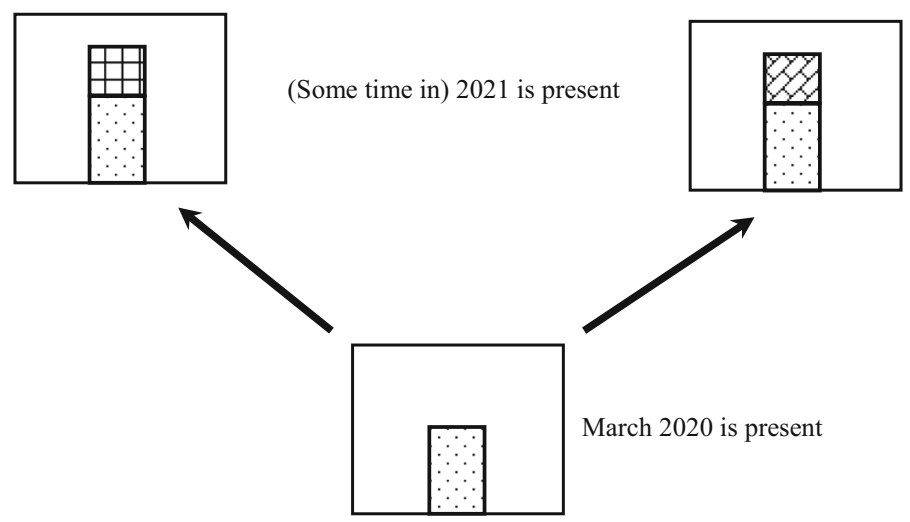

Fig. 1 Three possible stages and two ways the block can grow after having reached the stage in which March 2020 is present

is also a possible stage according to which March 2020 is present and which agrees with the first two stages on everything that happens in the world from the Big Bang up to March 2020. In this case, the first two possible stages (according to which 2021 is part of the block of reality) represent two possible future stages of the growth of the block with respect to the third stage (according to which March 2020 is present). In other words, when the third stage is actual, the first two stages represent two possible ways the block can grow in the future: by incorporating a 2021 in which the pandemic is still raging or by incorporating a 2021 in which the pandemic is over (see Fig. 1). The central idea of this paper can thus be formulated as the idea that supervaluationist growing-block theorists should take Thomason's times to be entities that represent possible stages of the growth of the growing-block of reality.

As I have suggested elsewhere (Loss, 2019: pp. 1150-1151), it seems possible to use this kind of stage-talk to express the general idea that temporal passage is real, and namely, by saying that there are at least two different stages of temporal passage such that at one stage reality is in a certain way and at some later stage reality is in a different way, incompatible with the first. For instance, moving-spotlight theorists (who take present, past, and future entities to all exist) may claim that there is a stage at which the time that instantiates the fundamental property of presentness is, say, T1 and a later stage at which the time that instantiates the fundamental property of presentness is T2; presentists (according to which only present entities exist and presently instantiate properties and relations) can say that there is a stage at which only certain present entities exist presently instantiating certain properties and relations and also a later stage at which different present entities instantiate different properties and relations; finally, as we just discussed, growing-block theorists may claim instead that there is a stage at which the latest time on the block of reality is (say) T1 and a later stage at which the latest time on the block of reality is instead T2.

A precursor of this talk of 'stages' can be found in McCall (1984) when he discusses his shrinking tree theory of time (according to which the universe is like a tree of historic possibilities branching towards the future, and the passage of time consists in the progressive loss of the 'lower branches' of reality): 
Instead of a different tree for each time, we can think of the different trees as 'snapshots' of the universe at different times. The snapshots look different, showing that time is passing and the universe is growing older. [...] the fourdimensional universe has more branches at an earlier stage and fewer branches at a later stage [...]' (McCall, 1984: p. 175; my italics).

McCall's idea may be expressed more in general as follows. Suppose that the universe is A-theoretic and imagine that God keeps taking pictures of the whole of reality as time goes by. What all these pictures will depict will depend on which A-theory is true in this universe: if presentism is true, every picture will depict only present entities presently instantiating properties and relations; if the moving spotlight theory is true, then while every picture will depict the same block of reality, spanning, say, from the Big Bang to the Big Crunch, each picture will represent the 'spotlight' of presentness as 'illuminating' a different time; instead, if the growing-block theory is true, the pictures of the whole of reality taken by God will each represent reality as spanning from the Big Bang up to a different time in history (see Fig. 2). In all of these cases, each picture represents a stage of temporal passage-a moment in the process consisting in the kind of change temporal passage amounts to according to the A-theory at hand (namely: a change in what present entities exist and what properties and relations they instantiate for presentists; a change in which time instantiates the property of 'being present' for moving-spotlight theorists; a change in which time is the 'edge of reality' for growing-block theorists). The general thought behind this way of understanding the reality of temporal passage doesn't seem to be too controversial, as it is witnessed by the very common use of figures resembling Figure 2 in the literature on dynamic theories of time. To mention just a few examples, consider Smith's (2011: pp. 236-237) Figs. 3 and 4, Leininger's (2015: pp. 726-767) Figs. 1 and 2, and Baron and Miller's (2019: pp. 21, 23-24) Figs. 2, 3, and 4. All these figures are naturally taken to represent how the various stages of temporal passage look like (and differ from one another) according to the various A-theories under consideration.

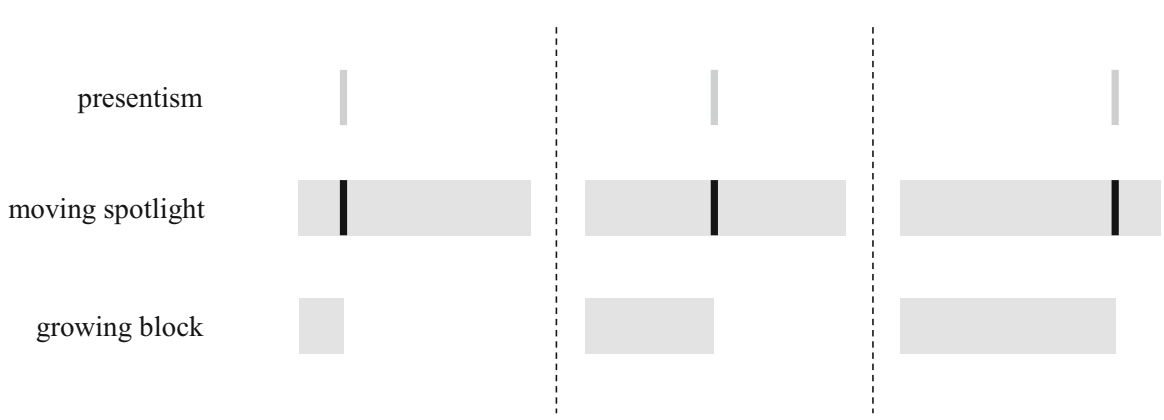

Fig. 2 Three stages of temporal passage according to presentism, the moving-spotlight theory, and the growing-block theory 

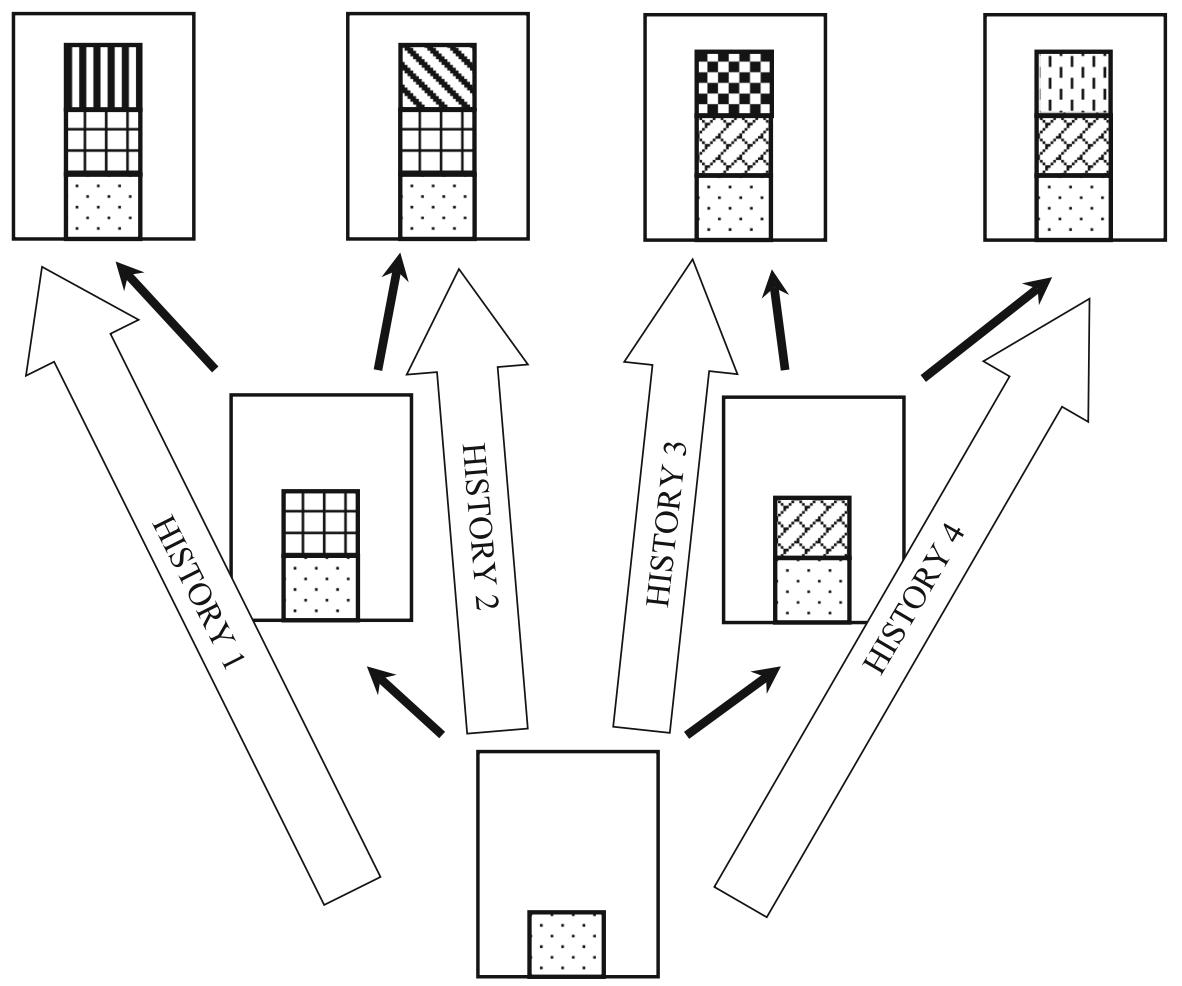

Fig. 3 A branching tree featuring four histories. Each history comprises three stages. In every history we have that, first (= at the first stage on that history), only one time exists; then (= at the second stage on that history), two times exist, and then (= at the third stage on that history) three times exist

It is very important to stress that this 'stage-theoretical' way of expressing the reality of temporal passage does not assume that stages are some kind of fundamental entities to which A-theorists are ontologically committed. As a matter of fact, at least in principle stage-talk may be reduced to either times, ersatz times ${ }^{13}$ hyper-times, ${ }^{14}$ ersatz hyper-times, or even to 'fragments of reality' (in the sense of Fine's, 2005 'fragmentalism'). What is important is only that dynamic theorists of time be able to make sense of this kind of talk, regardless of how they take it to be reducible. For instance, as indicated in Sect. 2.2, in this paper I will take growing-block theorists (qua A-theorists ${ }^{15}$ to make use of some irreducible, fundamental, and joint-carving tense operators to express truths like 'some $x$ is such that it was never the case that $x$ exists'

\footnotetext{
13 On ersatz times see, among others, Bourne (2006: pp. 52-61) and Crisp (2007: pp. 98-105).

14 See Skow (2015: pp. 44-49) for a recent example of the use of hyper-times (or 'super-times') in connection with dynamic theories of time.

15 To be more precise, I am considering here a 'standard' version of the growing-block theory in the sense of what Fine (2005) calls 'standard tense realism'. Elsewhere (Loss 2019: pp. 1151-1152) I provide a stagetheoretical characterisation of Fine's standard realism as the theory according to which reality is oriented towards one particular stage of temporal passage (which I am calling here the 'actual' one).
} 
and 'everything will always exist'. Therefore, in this paper I am assuming that stagetalk is reducible to the more fundamental tensed talk expressing truths concerning the growth of the block. This means that the stage-theoretical semantics I will present below is not a reductive one. However, as Krämer (2021) (echoing Fine, 2012: p. 2) efficaciously points out (while discussing his non-reductive truthmaker semantics for ground),

[t]here are a number of important uses for a formal semantics which do not depend on its reductive potential. [...] A formal semantics provides the resources to define relations of consequence $[\ldots]$ and consistency [...]. It thereby allows us to evaluate proposed deductive systems for the relevant languages for soundness and completeness. It may also allow us to connect the question of the consistency of a particular set of [...] claims with specific conditions on models. We can then see what impact it has on the logic if we allow or disallow such models, and thereby obtain further evidence for or against the consistency of the [...] claims in question. (Krämer, 2021: p. 12)

It should, thus, be clear that, even if non-reductive, the kind of semantics I will present below is not only not devoid of philosophical interest but can actually provide growingblock theorists with a powerful way to establish the overall logical and philosophical stability of their theory.

\section{Thomason's (1970) 'times' as stages}

As stated in the previous section, I will take here Thomason's (1970) 'times' to be ersatz possible worlds representing all the possible stages of the growth of the block. For simplicity's sake from now on I will simply call these ersatz worlds 'stages'. I will take the set of stages (in this sense) as being ordered in a tree-like fashion by a temporal relation $R .{ }^{16}$ When a stage $m$ and a stage $n$ are such that $m R n$ this means that the possible stage represented by $m$ could be followed by the possible stage represented by $n$. Any maximal linear chain of stages-any history-represents thus a possible way the block may grow as time goes by (see Fig. 3). ${ }^{17}$ As anticipated in Sect. 2.1, this paper focuses mainly on the way the ontology of a growing-block universe 'grows' as time passes. For this reason, in what follows I will focus only on a few necessary conditions that any growing-block theory of the kind discussed in this paper should plausibly accept and leave open the more general issue about what are the necessary and sufficient conditions for a stage to be followed by another stage. Consider, for instance, the following constraint on $R$ (where, for every moment $m, D(m)$ is the set of entities that exist according to $m$ ):

(D) For every $m$ and $n$, if $m R n$, then $D(m) \subset D(n)^{18}$

\footnotetext{
16 I say that $R$ is a 'temporal' relation only in the sense that if a stage $m$ is $R$-related to $n$, this means that $m$ is represented as preceding $n$ (while there is no actual temporal relation of precedence holding between the two abstract entities $m$ and $n$ ). 'Quasi-temporal' may be a more precise term. In what follows I will stick to 'temporal' for simplicity's sake.

17 Here and throughout the paper I am ignoring the possibility that the growing-block might stop growing. See on this Briggs and Forbes (2012: pp. 268-270), Correia and Rosenkranz (2018: p. 111) and Loss (2019). 18 This is the same condition Correia and Rosenkranz (2018: p. 168) call '(p1)'.
} 
According to (D), a stage $n$ can be $R$-later than a stage $m$ only if the domain of $m$ is a proper subset of the domain of $n$. In other words, all the entities that exist at $m$ must exist at $n$, and some new entity must exist at $n$ that didn't exist at $m$. (D) appears to encapsulate the very ontological core of the growing-block theory as expressed by (BR1)-(BR2), and (GB1)-(GB2) (see Sect. 1): as time goes by, no entity ever ceases to exist ('it is always the case that every $x$ is such that $x$ will always exists'), and newer and newer entities keep coming into existence ('it is always the case that some $x$ is such that $x$ never existed before').

Further relatively uncontroversial constraints on $R$ concern times and their temporal ordering. Letting, for every stage $m, T(m)$ be the set of times existing at $m$-plausibly, a subset of $D(m)$ — and ' $\prec$ ' a linear temporal relation of precedence among times (holding with respect to stages), these necessary conditions can be presented as follows:

(T1) For every stages $m$ and $n$, if $m R n$, then $T(m) \subset T(n)$

(T2) For every stages $m$ and $n$, and times $t$ and $s$ such that $t \in T(m), s \in T(m)$, if $m R n$, then $t \prec s$ according to $m$ if and only if $t \prec s$ according to $n$

(T3) For every stage $m$ there is a unique time $t \in T(m)$ such that every other time $s \in T(m)$ is such that, according to $m, s \prec t$

According to (T1), every time keeps existing (as a time) at later stages of temporal passage. According to (T2) the temporal ordering of previously existing times doesn't change as time goes by. Finally, (T3) requires that every stage features a time that is the latest time on the block of reality. (T1), (T2) and (T3) appear to further specify in a plausible way how the block grows for growing-block theorists. ${ }^{19}$

\section{The growth of the block}

Above we have considered the semantic clauses for Boolean connectives and tense operators. Growing-block theorists interpreting supervaluationism stage-theoretically can simply adopt them by replacing times with stages. This means, for instance, that 'It will be the case that $p$ ' can be taken to be true at a $<$ stage, history $>$ pair $\langle m, h\rangle$ if and only if there is some stage $n$ belonging to $h$ that is $R$-later than $m$ and such that $p$ is true at $\langle n, h\rangle$, and 'It was the case that $p$ ' can be taken to be true at a $<$ stage, history $>$ pair $\langle m, h\rangle$ if and only if there is some stage $n$ belonging to $h$ that is $R$-earlier than $m$ and such that $p$ is true at $\langle n, h\rangle$ (in what follows $M$ is the set of all stages):

$$
\begin{aligned}
& \langle m, h\rangle \vDash F p \Leftrightarrow \exists n[n \in M \wedge m R n \wedge n \in h \wedge\langle n, h\rangle \vDash p] \\
& \langle m, h\rangle \vDash P p \Leftrightarrow \exists n[n \in M \wedge n R m \wedge n \in h \wedge\langle n, h\rangle \vDash p]
\end{aligned}
$$

Moving on to monadic predicates and quantification, we can add to our theory the following two clauses (where ' $<m, h>\vDash_{s} \phi$ ' stands here for $\phi$ is true with respect to the stage $m$ and history $h$ under the assignment $s$, where an assignment is a function from the set of variables to the set $D$ of entities that exist at some possible stage or

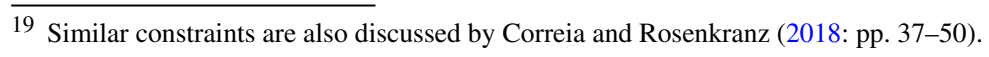


other, ' $d e n_{s}(a)$ ' is the denotation of an individual term $a$ on a given assignment $s$, ' $s[x / d]$ ' is an assignment that only differs from $s$ by assigning to the variable ' $x$ ' the object $d$, and ' $I_{m}$ ' is a function from the set of monadic predicates to the power set of $D(m)): 20,21$

$$
\begin{gathered}
\langle m, h\rangle \models_{s} F a \Leftrightarrow \operatorname{den}_{s}(a) \in I_{m}(F) \\
\langle m, h\rangle \models_{s} \exists x \phi \Leftrightarrow \text { for some } d \in D(m):\langle m, h\rangle \models_{s[x / d]} \phi
\end{gathered}
$$

The clause for the existential quantifier is the most important for us here. In fact, we are assuming not only that every possible stage of the growth has multiple possible futures, but also that in order for a stage to count as $R$-later than another stage its domain must be a proper superset of the domain of the latter. It is then easy to check that (GB1) and (GB2) come out valid in our theory (that is, super-true at every possible stage):

$$
\begin{aligned}
& \text { (GB1) } A \exists x \sim P \mathbb{E} x \\
& \text { (GB2) } A \forall x G \mathbb{E} x
\end{aligned}
$$

Furthermore, given (T1), (T2), and (T3) we also have the validity of the following statements concerning times and presentness (letting ' $\pi(t)$ ' stand for ' $t$ is present' and defining it as follows: $\left.\pi(t)={ }_{d f} \forall y((T y \wedge y \neq t) \rightarrow y \prec t)\right)$ :

$$
\begin{array}{ll}
\text { (GB3) } & A \exists x(T x \wedge H \sim \mathbb{E} x) \\
\text { (GB4) } & A \forall x(T x \rightarrow G(\mathbb{E} x \wedge T x)) \\
\text { (GB5) } & A \forall x \forall y((T x \wedge T y \wedge x \prec y) \rightarrow G(x \prec y)) \\
\text { (GB6) } & A \exists ! x(T x \wedge \pi(x) \wedge H \sim \mathbb{E} x)
\end{array}
$$

The validity of (GB1)-(GB6) is clearly a much welcome result for growing-block theorists.

\section{Correia and Rosenkranz's (2018) objection}

In this paper I am assuming a version of the growing-block theory according to which future-contingent statements are neither true nor false. The idea that growing-block theorists are committed to such a view is not uncontroversial. For instance, Correia and Rosenkranz (2018: ch. 7) have defended the idea that growing-block theorists can

\footnotetext{
20 Here I am loosely drawing on the presentation of Kripke semantics for first-order modal logic given by Williamson (2013: pp. 119-22). I focus only on monadic predicates for simplicity's sake.

21 The relation between $D$ and all the sets $D(m)$ is, thus, the following:
}

$$
(*) \forall x(x \in D \leftrightarrow \exists m(m \in M \wedge x \in D(m))
$$


uphold both the validity of the principle of bivalence for future-contingent statements and the idea that truth is (somehow) grounded in reality. As a part of their argument in favor of bivalence in the open future Correia and Rosenkranz (2018: p. 105) criticize the supervaluationist account of future-contingent statements as incompatible with the central tenets of the growing-block theory. It may be useful to quote the relevant passage in full:

According to supervaluationism, $\varphi$ is true at a time $t$ just in case $\varphi$ is supertrue at $t$; and $\varphi$ is false at time $t$ just in case $\varphi$ is superfalse at $t$. Supertruth, and superfalsity, at a given time $t$ are defined as follows: $\varphi$ is supertrue at time $\boldsymbol{t}$ just in case $\varphi$ is true at ton all histories that include $t$; and $\varphi$ is superfalse at time $t$ just in case $\varphi$ is false at $t$ on all histories that include $t$. Here, a history is a maximal linear set of times $[\ldots]$.

[...] it should be clear that the supervaluationist treatment cannot be made to square with one of the consequences of [the growing-block theory], viz. that there is no time later than now. For if now is the last time, there can be only one history, that history having a last moment, namely now. Accordingly, given the supervaluationist's semantic treatment of the future tense operator $F$ and of the operators $\neg$ and \&, all statements of type $\neg F \varphi \& \neg F \neg \varphi$ must be taken to be supertrue now, and hence to be true now. This means that given the supervaluationist view, [the growing-block theory] is committed to time having just come to an end. This is, of course, an unfortunate result. (Correia \& Rosenkranz, 2018: p. 105; bold mine).

According to the version of the growing-block theory defended in this paper, stages and times are different kind entities playing different roles. At every stage of the growth of the block that is later than the very first stage (if any), there exist many different times, linearly ordered by the earlier-later relation $\prec$, and such that one of them is the last time on the block of reality. Therefore, it is indeed true, according to version of the growing-block theory presented above, that at the current ('actual') stage of the growth of the block there is one time that is the last existing time. From this, however, it doesn't follow that 'there can be only one history' having the time that is now present (that is, the last time on the block) has its last time. In fact, according to this version of supervaluationism, histories are maximal linear chains of stages and the supervaluationist notion of super-truth is taken to be relative to stages, rather than times. Therefore, the fact that at the current stage of temporal passage there is one time $t$ that is the 'edge of reality' is perfectly compatible with the existence of multiple histories each featuring a different way in which the block can grow in the future and all agreeing on the fact that, as the block grows, $t$ ceases to be the last time on the block and becomes, in this sense, 'past'.

We can, thus, conclude that the stage-theoretical account of supervaluationism presented in this paper shows that, pace Correia and Rosenkranz (2018: p. 105), supervaluationism is perfectly compatible with the main claims of the growing-block theory of time. 


\section{Briggs and Forbes's (2012) 'quantifier troubles' (and how to avoid them)}

Briggs and Forbes (2012) have presented what may be the most prominent supervaluationist version of the growing-block theory in recent literature. Their theory is built using ersatz times, each of which is endowed with its own domain of entities. Timelines are 'sets of ersatz times ordered by a total, transitive, irreflexive, and asymmetric relation $[L]$ ' (Briggs \& Forbes, 2012: p. 260; I have labelled ' $L$ ' the temporal relation they label ' $R$ ' not to confuse it with our $R$-relation; see above). As Briggs and Forbes point out:

There is one timeline that represents the world as it really is - that is, it correctly represents all the times that concretely exist, and it does not represent the world as containing any times that don't concretely exist. This is the actualized timeline.

(Briggs \& Forbes, 2012: p. 264)

Briggs and Forbes take a history to be a 'maximal set of times $h$ such that for any two distinct times in the set, one bears the $[L]$ relation to the other. (One can picture histories as branches of the tree stretching all the way from root to leaf-tip.)' (Briggs \& Forbes, 2012: p. 277). Simplifying a bit (and ignoring for simplicity's sake the complications that arise by supposing that time may come to an end), ${ }^{22}$ Briggs and Forbes's version of supervaluationism can be presented (and reformulated using the notation employed in this paper) as follows. The semantic clauses for Boolean operators, quantifiers, and temporal operators are given in terms of the notion of truth with respect to a <ersatz time, history> pair (see Briggs \& Forbes, 2012: p. 279) (in what follows I will focus only on temporal operators and Briggs and Forbes's 'tenseless' quantifier ' $\Sigma x$ ', ${ }^{23}$ where $D(e)$ is the domain of entities that exist at the ersatz time $e)^{24}$ :

$$
\begin{gathered}
\langle e, h\rangle \vDash_{S} F p \Leftrightarrow \exists e^{\prime}\left[e L e^{\prime} \wedge e^{\prime} \in h \wedge\left\langle e^{\prime}, h\right\rangle \vDash p\right] \\
\langle e, h\rangle \models_{s} P p \Leftrightarrow \exists e^{\prime}\left[e^{\prime} L e \wedge e^{\prime} \in h \wedge\left\langle e^{\prime}, h\right\rangle \vDash p\right] \\
\langle e, h\rangle \vDash_{s} \Sigma x \phi \Leftrightarrow \exists e^{\prime} \exists d\left[e^{\prime} \in h \wedge d \in D\left(e^{\prime}\right):\left\langle e^{\prime}, h\right\rangle \vDash_{s[x / d]} \phi\right]
\end{gathered}
$$

The notions of super-truth and super-falsity are defined with respect to timelines as follows:

\footnotetext{
22 See Briggs and Forbes (2012: pp. 268-70).

${ }^{23}$ Briggs and Forbes (2012: p. 262) also define a 'tensed' existential quantifier that quantifies only over entities existing relative to a given ersatz time as follows:

$$
<e, h>\models_{s} \operatorname{Ex} \phi \Leftrightarrow \exists d\left[d \in D(e):<e, h>\models_{s[x / d]} \phi\right]
$$

In what follows I will mainly ignore this quantifier for simplicity's sake (see, however, footnote 31 ).

24 Notice that, in order to be consistent with the rest of the paper I define here their tenseless quantifier ' $\Sigma x$ ' as an objectual quantifier rather than a substitutional one (Briggs and Forbes 2012: p. 262). Given the purpose of this paper, nothing of substance hangs on this.
} 
$S$ is super-true with respect to a timeline $T$ if and only if every history $h$ containing $T$ (and, thus, 'maximally extending' it) is such that $S$ is true with respect to $\left\langle e_{T}, h\right\rangle$, where $e_{T}$ is the last time of $T$ (i.e. its present);

$S$ is super-false with respect to a timeline $T$ if and only if every history $h$ containing $T$ is such that $S$ is false with respect to $\left\langle e_{T}, h\right\rangle$; otherwise, $S$ is neither true nor false at $T .^{25}$

As Briggs and Forbes (2012: pp. 293-5) notice, their account appears to suffer from an important problem. In fact, according to their theory, the existential quantifier ' $\Sigma$ ' ranges over every entity that exists at some time or other with respect to a given history. This means that if in every possible future of a certain timeline $T$ a certain entity $a$ exists, the sentence ' $\Sigma x(x=a)$ ' (saying that $a$ exists) turns out to be supertrue at $T$ even if there is no time $e$ in $T$ such that $a$ exists at $e$ (that is, even if there is no time $e$ in $T$ such that $a \in D(e)$ ). Therefore, according to this theory it is false that only past and present entities exist, given that $a$ exists even if there is no past or present time at which it exists. Briggs and Forbes claim that this problem only shows that 'ontological commitment cannot be read off the tenseless existential quantifier $\Sigma$ ' (Briggs \& Forbes, 2012: p. 294). Instead, they introduce for this purpose the timelineindexed quantifier ' $\Sigma_{T}$ ' along the following lines ${ }^{26}$ :

$$
\langle e, h\rangle \models_{s} \Sigma_{T} x \phi \Leftrightarrow \exists e^{\prime} \exists d\left[e^{\prime} \in T \wedge d \in D\left(e^{\prime}\right):\left\langle e^{\prime}, h\right\rangle \models_{s[x / d]} \phi\right]
$$

The idea is that the existential quantifier that growing-block theorists can use to express their ontological commitments should be a quantifier that restricts its attention only to the actualized timeline, as it were, thus excluding all the entities that are represented as existing according to 'possibly future' ersatz times. Even if we assume that in every possible future of the timeline $T$ a certain entity $a$ exists, the sentence ' $\Sigma_{T} x(x=a)$ ' turns out to be super-false at $T$ if there is no time $e$ in $T$ such that $a$ exists at $e$. This is indeed a desideratum of the growing-block theory of time, given that one of its main tenets is indeed that (merely) future entities don't exist. Notice, however, that other central claims of the growing-block theory include claims like (GB1) and (GB2) saying and entailing that there are things that didn't exist in the past and that in the future there will be things that will exist then but don't exist now. It is then easy to check that, for every timeline $T$, the following two claims are super-false at $T$ according to Briggs and Forbes's theory (where ' $F_{n}$ ' and ' $P_{n}$ ' are the metric tense-operators 'it will

\footnotetext{
25 Notice that Briggs and Forbes (2012: p. 280) actually define super-truth and super-falsity with respect to both an ersatz time and a timeline. However, the definitions given here appear to follow from their definition of absolute truth at a timeline: 'what is true at a timeline $T$ is simply what is true at $T$ 's absolute present', where the '(ersatz) absolute present' of a timeline is 'the last time in it' (Briggs and Forbes 2012: p. 264).

26 Briggs and Forbes (2012: p. 294) present a general definition of ' $\Sigma_{T}$ ' by using the notion of truth at an ersatz time and timeline. However, it seems clear that supervaluationists must define ' $\Sigma_{T}$ ' in terms of the notion of truth at an ersatz time and a history (given the way they define the notion of super-truth). Notice, however, that since what is important here is that ' $\Sigma_{T}$ ' quantifies only over entities existing according to the timeline $T$, so to speak, this difference is irrelevant for present purposes.
} 
be the case $n$ time-units hence' and 'it was the case $n$ time units ago'): ${ }^{27,} 28$

$$
\begin{aligned}
& (\mathrm{GB}-\mathrm{BF} 1) \quad \Sigma_{T} x \sim P \Sigma_{T} y(x=y) \\
& (\mathrm{GB}-\mathrm{BF} 2) F_{n} \Sigma_{T} \sim P_{n} \Sigma_{T} y(x=y)
\end{aligned}
$$

As a matter of fact, not only ' $\Sigma_{T}$ ' is a rigid quantifier that is always restricted to the times that belongs to the timeline $T$, but Briggs and Forbes's temporal operators only shift the ersatz time of evaluation, whereas intuitively the past and future growth of the block can be appreciated only by comparing the current actualized timeline with the timelines that were actualized and the timeline that could be actualized in the future.

It should be clear at this point that Briggs and Forbes's timelines can be seen (mutatis mutandis) as the counterparts of our stages in their theory, each representing a possible stage of the growth of the growing block. Briggs and Forbes also consider a relation of 'feasibility' between timelines (Briggs \& Forbes, 2012: pp. 267-270) that seems to play the same role of our $R$-relation between stages. Indeed, Briggs and Forbes's theory can be made relevantly similar to the theory presented in this paper as follows. First, the notion of 'T-history' is introduced by saying that a T-history is a maximal linear chain of timelines linearly ordered by the feasibility relation. Second, the temporal operators ' $\mathbf{F}$ ' and 'P' are defined in terms of the notion of truth a <ersatz time, timeline, T-history > triple and understood as capable of shifting the timeline parameter along a T-history as follows (where ' $f$ ' is the feasibility relation):

$$
\begin{gathered}
\langle e, T, H\rangle \vDash \mathbf{F} p \Leftrightarrow \exists T^{\prime}\left[T f T^{\prime} \wedge T^{\prime} \in H \wedge\left\langle e, T^{\prime}, H\right\rangle \vDash p\right] \\
\langle e, T, H\rangle \vDash \mathbf{P} p \Leftrightarrow \exists T^{\prime}\left[T^{\prime} f T \wedge T^{\prime} \in H \wedge\left\langle e, T^{\prime}, H\right\rangle \vDash p\right]
\end{gathered}
$$

27 ' $F_{n}$ ' and ' $P_{n}$ ' can be defined within Briggs and Forbes's theory just like they define ' $F$ ' and ' $P$ ' but adding that the relevant future/past ersatz time must be at the right temporal distance from the ersatz time in question. For instance, one could add to the theory a function 'dist' from pairs of ersatz times to the set of natural numbers (assuming for simplicity's sake that temporal passage is discrete) and define ' $F_{n}$ ' and ' $P_{n}$ ' as follows:

$$
\begin{gathered}
<e, h>\models F_{n} p \Leftrightarrow \exists e^{\prime}\left[e e^{\prime} \wedge e^{\prime} \in h \wedge \operatorname{dist}\left(e, e^{\prime}\right)=n \wedge<n, h>\models p\right] \\
<e, h>\models P_{n} p \Leftrightarrow \exists e^{\prime}\left[e^{\prime} \operatorname{Re} \wedge \operatorname{dist}\left(e, e^{\prime}\right)=n \wedge<e^{\prime}, h>\models p\right]
\end{gathered}
$$

28 A claim along the lines of (GB-BF2) could be expressed by means of Briggs and Forbes's tensed quantifier (see footnote 26) as follows:

$$
\left(\mathrm{GB}-\mathrm{BF} 2^{*}\right) F_{n} \mathrm{Ex} \sim P_{n} \mathrm{E} y(x=y)
$$

Notice, however, that even using tensed quantifiers it appears difficult for Briggs and Forbes's theory to express claims like the following:

(C*) It will be the case $n$ time units hence that there (tenselessly) is an $x$ that is (i) not present and also such that (ii) it was the case $n$ time units ago that $x$ doesn't (tenselessly) exists. 
Third, a quantifier ' $\mathcal{E}$ ' is defined as being sensitive to what exists at a given timeline but without being rigidly bound to any given timeline:

$$
\langle e, T, H\rangle \vDash_{s} \mathcal{E} x \phi \Leftrightarrow \exists e^{\prime} \exists d\left[e^{\prime} \in T \wedge d \in D\left(e^{\prime}\right):\langle e, T, H\rangle \vDash_{s[x / d]} \phi\right] ;
$$

Finally, the notion of super-truth and super-falsity at a timeline is defined as follows:

$S$ is super-true with respect to a timeline $T$ if and only if every T-history $H$ containing $T$ is such that $S$ is true with respect to $\left\langle e_{T}, T, H\right\rangle$;

$S$ is super-false with respect to a timeline $T$ if and only if every T-history $H$ containing $T$ is such that $S$ is false with respect to $\left\langle e_{T}, T, H\right\rangle$; otherwise, $S$ is neither super-true nor super-false at $T$.

It can be easily checked that this new extension of Briggs and Forbes's theory predicts both that ' $\mathcal{E} x(a=x)$ ' is false for any merely future entity $a$ whose future existence is settled and also that the following claims are true ${ }^{29}$ :

$$
\begin{aligned}
& (\mathrm{GB}-\mathrm{BF}-\mathrm{N} 1) \quad \mathcal{E} x \sim \mathbf{P} \mathcal{E} y(x=y) \\
& (\mathrm{GB}-\mathrm{BF}-\mathrm{N} 2) \quad \mathbf{F}_{n} \mathcal{E} x \sim \mathbf{P}_{n} \mathcal{E} y(x=y)
\end{aligned}
$$

However, this point just helps consolidate the central claim of this paper. In fact, what we have done here is just to individuate the entities that can count as stages in Briggs and Forbes's theory and then build a new theory around the notion of super-truth and super-falsity relative to a stage by taking histories to be linear chain of stages (instead of times). In other words, we have interpreted Briggs and Forbes's theory stage-theoretically, which is precisely what this paper argues we should do when dealing with supervaluationist accounts of the growing-block theory of time. ${ }^{30}$

\footnotetext{
29 ' $\mathbf{F}_{n}$ ' and ' $\mathbf{P}_{n}$ ' can be defined as ' $F_{n}$ ' and ' $P_{n}$ ' but using instead a distance function for timelines instead of times.

30 This modified version of Briggs and Forbes's theory is still different from the supervaluationist account presented above. Most importantly, this version of Briggs and Forbes's theory still treats ersatz times as part of the 'indices of evaluation' thus allowing the existence of 'weak' (Loss 2019: p. 1148) tense operators capable of shifting the time-parameter of the indices of evaluation (that is, ' $P$ ' and ' $F$ ' above) alongside the 'strong' tense operators capable of shifting the timeline-parameter (that is, ' $\mathbf{P}$ ' and ' $\mathbf{F}$ ' above). A thorough comparison between these two theories is beyond the scope of this paper. Notice, however, that although growing-block theorists can indeed understand and accept the existence of a 'weak' form of tense in the sense just specified, at least from a metaphysical point of view they should only regard as irreducible the kind of 'strong' tense they employ to express the growth of the block. Therefore, a language featuring only strong tense operators and expressing 'weakly tensed' truths only by means of quantification over times appears to be more 'metaphysically perspicuous' and 'joint-carving' then one also employing weak tense operators.
} 


\section{Conclusion}

It may seem natural for growing-block theorists to reject the principle of bivalence for future-contingent statements. At the same time, Thomason's (1970) supervaluationism may strike many as the best semantic treatment of truth-value gaps in the open future. In this paper I haven't defended either of these two claims. However, I have argued that growing-block theorists who agree with them should embrace a stage-theoretical interpretation of supervaluationism according to which Thomason's possible histories aren't constructed out of possible times, but rather out of possible stages of the growth of the growing block. As I have shown, this way of construing the supervaluationist machinery is not only perfectly compatible with the main tenets of the growing-block theory (pace Correia \& Rosenkranz, 2018: p. 105), but also avoids the problems concerning quantification that threaten Briggs and Forbes's (2012) version of supervaluationism. ${ }^{31}$

\section{References}

Baron, S., \& Miller, K. (2018). An Introduction to the Philosophy of Time. Polity Press.

Belnap, N. (2009). Truth values, neither-true-nor-false, and supervaluations. Studia Logica, 91(3), 305-334.

Bourne, C. (2002). When Am I? A Tense Time for Some Tense Theorists? Australasian Journal of Philosophy, 80, 359-371.

Braddon-Mitchell, D. (2004). How Do We Know It is Now Now? Analysis, 64, 199-203.

Braddon-Mitchell, D. (2013) Fighting the zombie of the growing Salami, in Karen Bennett \& Dean Zimmerman (eds.), Oxford Studies in Metaphysics (Vol. 8, pp. 351-361), Oxford University Press.

Briggs, R., \& Forbes, G. A. (2012) The real truth about the unreal future. In Bennett, K., Zimmerman, D (eds.) Oxford Studies in Metaphysics (Vol. 7, pp. 257-304). Oxford University Press.

Broad, C. D. (1923). Scientific Thought. Routledge and Kegan Paul.

Bourne, C. (2006). A Future for Presentism. Oxford University Press.

Cameron, R. (2018). Skow, objective becoming and the moving spotlight. Analysis, 78(1), 97-108.

Correia, F., \& Rosenkranz, S. (2013) Living on the brink, or welcome back, Growing Block! In Bennett, K., Zimmerman, D. (Eds.) Oxford Studies in Metaphysics (Vol. 8, pp. 333-350). Oxford University Press.

Correia, F., \& Rosenkranz, S. (2015). Presentism without presentness. Thought: A Journal of Philosophy, 4(1), 19-27.

Correia, F., \& Rosenkranz, S. (2018). Nothing to come: A defence of the growing block theory of time. Synthese Library.

Crisp, T. (2007). Presentism and the grounding objection. Noûs, 41, 118-137.

Deasy, D. (2017). What is Presentism? Noûs, 51(2), 378-397.

Fine, K. (2005). Modality and Tense. Oxford University Press.

Fine, K. (2012). The pure logic of ground. The Review of Symbolic Logic, 5, 1-25.

Forbes, G. (2016). The Growing Block's past problems. Philosophical Studies, 173, 699-709.

Forrest, P. (2004). The real but dead past: A reply to Braddon-Mitchell. Analysis, 64(4), 358-362.

Goranko, V. and A. Rumberg (2020). Temporal Logic. In Edward N. Zalta (ed.) The Stanford Encyclopedia of Philosophy (Summer 2020 Edition). https://plato.stanford.edu/archives/sum2020/entries/logictemporal/. Last accessed July 4, 2021.

Grandjean, V. (2019). How is the asymmetry between the open future and the fixed past to be characterized? Synthese. https://doi.org/10.1007/s11229-019-02164-2

Hawley, K. (2001). How Things Persist. Oxford University Press.

\footnotetext{
31 Work on this article has received funding from the Deutsche Forschungsgemeinschaft (project number 448954791) and the Spanish Ministerio de Ciencia e Innovación (grant number: RYC2019-026416-I). Many thanks to two anonymous referees for this Journal for comments that helped improve the paper.
} 
Krämer, S. (2021). Singular troubles with singleton Socrates. Philosophy and Phenomenological Research. https://doi.org/10.1111/phpr.12692

Leininger, L. (2015). Presentism and the Myth of Passage. Australasian Journal of Philosophy, 93(4), 724-739.

Loss, R. (2019). No ground for doomsday. Inquiry: an Interdisciplinary Journal of Philosophy, 62(9-10), $1136-1156$.

McCall, S. (1984). A dynamic model of temporal becoming. Analysis, 44(4), 172.

Menzel, C. (2017). Possible Worlds. The Stanford Encyclopedia of Philosophy (Winter 2017 Edition), Edward N. Zalta (ed.). https://plato.stanford.edu/archives/win2017/entries/possible-worlds/.

Merricks, T. (2006). Goodbye growing block. In Zimmerman, D. (Ed.) Oxford Studies in Metaphysics (Vol. 2, 103-110). Oxford University Press.

Pooley, O. (2013). Relativity, the Open Future, and the Passage of Time. Proceedings of the Aristotelian Society, 113, 321-363.

Sider, T. (2001). Four Dimensionalism. Oxford University Press.

Sider, T. (2011). Writing the Book of the World. Oxford University Press.

Skow, B. (2015). Objective Becoming. Oxford University Press.

Smith, N. (2011). Inconsistency in the A-Theory. Philosophical Studies, 156, 231-247.

Sullivan, M. (2012). The minimal A-theory. Philosophical Studies, 158, 149-174.

Tallant, J., \& Ingram, D. (2015). Nefarious Presentism. Philosophical Quarterly, 65(260), 355-371.

Thomas, E. (2019). The Roots of C. D. Broad's Growing Block Theory of Time. Mind 128(510): 527-549.

Publisher's Note Springer Nature remains neutral with regard to jurisdictional claims in published maps and institutional affiliations. 